\title{
Hematoma subdural como complicación de analgesia epidural de parto. A propósito de dos casos
}

Veloso de Sousa R, Ortega López AB, Guillén Perales JF.

Hospital Universitario San Cecilio, Granada.

\section{Resumen}

Las hemorragias intracraneales son una complicación infrecuente pero potencialmente grave tras la punción dural asociada a anestesia neuroaxial. Las consecuencias pueden ser catastróficas, especialmente cuando hablamos de anestesia neuroaxial obstétrica en mujeres jóvenes y sanas. El diagnóstico puede ser difícil por la similitud de síntomas con la cefalea postpunción dural (CPPD), que es la complicación más frecuente tras una punción dural. Este trabajo tiene como objetivo, aumentar la conciencia de los profesionales ante la posibilidad de ocurrencia de este tipo de complicaciones ante una punción dural accidental.

\section{Introducción}

Las hemorragias intracraneales son una complicación infrecuente pero potencialmente grave tras la punción dural asociada a anestesia neuroaxial. Las consecuencias pueden ser catastróficas, especialmente cuando hablamos de anestesia neuroaxial obstétrica en mujeres jóvenes y sanas. El diagnóstico puede ser difícil por la similitud de síntomas con la cefalea postpunción dural (CPPD), que es la complicación más frecuente tras una punción dural. Este trabajo tiene como objetivo, aumentar la conciencia de los profesionales ante la posibilidad de ocurrencia de este tipo de complicaciones ante una punción dural accidental.

Tras la punción dural, la perdida de líquido cefalorraquídeo (LCR) puede desplazar la masa encefálica en sentido caudal, ejerciendo tracción sobre las venas subdurales intracraneales, pudiendo estas romperse y sangrar hacia el espacio subdural, produciéndose el hematoma subdural (HSD). Los síntomas producidos se relacionan con el efecto masa, desplazamiento de estructuras e irritación meníngea.

\section{Caso 1}

Mujer de 32 años de edad, primigesta a término, que ingresa por pródromos de parto. Sin antecedentes personales de interés y embarazo normoevolutivo. Solicita realización de técnica epidural para analgesia de parto, realizándose punción a nivel L3-L4, con aguja de Tuohy $18 \mathrm{G}$, técnica de perdida de resistencia con aire, comprobándose punción dural accidental $\mathrm{y}$, a consecuencia, se retira la aguja. Se repite la técnica a nivel L2-L3, con colocación exitosa del catéter, aspiración y dosis test negativas y sin que se observaran otras complicaciones durante el trabajo de parto.

Dentro de las primeras $12 \mathrm{~h}$ tras la punción accidental, inicia clínica de cefalea occipito-frontal, pulsátil, sin otra clínica neurológica asociada y ausencia de signos de irritación meníngea. El 
dolor mejora con tratamiento conservador y es dada de alta, dos días tras el parto, con tratamiento analgésico domiciliario.

Reingresa 5 días tras el alta por reagudización de la cefalea, refiriendo empeoramiento súbito que se presenta durante la micción, que no mejora con el tratamiento domiciliario establecido. La exploración de la paciente es anodina, sin focalidad neurológica, sin signos de irritación meníngea. Ante la ausencia de signos de alarma, se decide mantener tratamiento conservador con fluidoterapia, perfusión analgésica con metamizol y dexketoprofeno, corticoterapia con Hidrocortisona $100 \mathrm{mg} / 8 \mathrm{~h}$, opioides de rescate y se recomienda que ingiera bebidas con cafeína. La evolución en las siguientes $48 \mathrm{~h}$ es favorable, con mejora de la sintomatología y persistiendo una exploración neurológica normal y cifras tensionales normales.

Al tercer día de ingreso, comienza con anopsia en ojo derecho, visión borrosa en ojo izquierdo, persistencia de cefalea e hipertensión arterial (HTA) a lo que se sigue la aparición de hasta 3 episodios de crisis tónico-clónicas generalizadas con estado post-critico pero recuperación del nivel de conciencia. Se realiza TAC y angio-TAC craneal urgente, en el que se objetiva pequeño HSD y varias imágenes hipodensas que se informan como higromas, en probable relación con hipotensión de LCR, sin signos significativos de hipertensión intracraneal (HIC) (FIG 1 y 2).

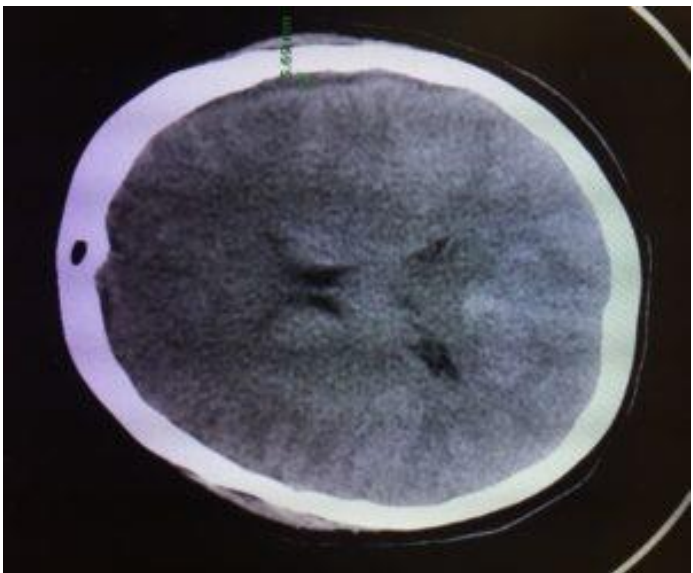

FIG 1: TAC craneal en el que se observa higroma parieto-temporal izquierdo.



FIG 2: TAC craneal muestra higroma pericelebeloso derecho

Dada la recurrencia de las crisis, se decide intubación orotraqueal y traslado a Unidad de Cuidados Intensivos (UCI) neurotraumatológicos.

Se controla la HTA con tratamiento i.v y se realiza Eco-doppler trascraneal en el que no se objetivan datos de HIC ni vasoespasmo.

Se procede a la extubación de la paciente a las $4 \mathrm{~h}$ de su ingreso en UCI. En los días siguientes, presenta evolución favorable, sin ocurrencia de nuevas crisis comiciales, manteniendo una exploración neurológica normal y control de HTA con tratamiento oral. En la TAC de control, realizado 5 días tras la TAC diagnóstica, se constata la práctica resolución de la hemorragia subaracnoidea y de los higromas subdurales. Se realiza también electroencefalograma donde se observa foco epileptógeno, por irritación. 
Es dada de alta 7 días tras el ingreso, con exploración neurológica normal.

\section{Caso 2}

Mujer de 27 años de edad, secundigesta a término, con antecedentes de parto instrumental hace 7 años, sin comorbilidades asociadas. Solicita analgesia epidural por parto espontáneo en curso. Se realiza punción dificultosa a nivel L3-L4, con aguja tipo Tuohy 18G, y técnica de perdida de resistencia con suero, con resultado de punción dural accidental, inyectándose inmediatamente $5 \mathrm{ml}$ de suero salino fisiológico a través de aguja de Tuohy. Se repite la técnica a nivel L2-L3, con colocación exitosa del catéter, aspiración $\mathrm{y}$ dosis test negativas. El parto trascurre sin nuevas incidencias.

La paciente refiere cefalea, de inicio en el postparto inmediato, que empeora con la bipedestación, sin focalidad neurológica asociada y ausencia de signos de irritación meníngea. El dolor se controla con tratamiento conservador y es dada de alta a domicilio con tratamiento analgésico y recomendación de hidratación abundante $\mathrm{y}$ movilización progresiva.

En domicilio, presenta empeoramiento progresivo de la cefalea, que le impide la realización de sus actividades normales, acudiendo al servicio de urgencias, a los 10 días tras el parto, por cefalea intensa.

La exploración neurológica en servicio de urgencias es normal, manteniendo puntuación de $15 / 15$ en la escala de Glasgow, destacando únicamente cefalea frontal y retroocular derecha intensas.

Se realiza TAC craneal (FIG 3), en la que se observa colección subdural derecha con pequeña hemorragia asociada, con importante efecto masa sobre línea media e incipiente herniación subfalciana. Los hallazgos sugieren hemorragia subdural aguda sobre higroma o HSD crónico.

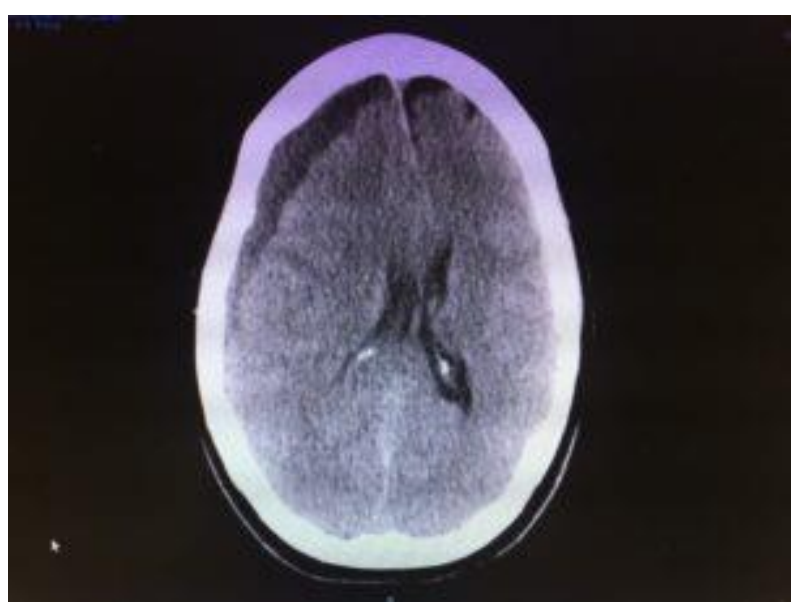

FIG 3: TAC de cráneo enseñando colección subdural derecha, con efecto masa y desviación de línea media.

Ante los hallazgos, se decide intervención quirúrgica urgente, con realización de trépano evacuador y lavado del hematoma epidural, bajo anestesia local y sedación. La intervención quirúrgica discurre sin incidencias desde el punto de vista anestésico y quirúrgico.

Durante el ingreso, la paciente presentó buena evolución clínica, con mejoría progresiva de la cefalea y persistiendo exploración neurológica normal. Es dada de alta 9 días tras el ingreso.

\section{Discusión}

La verdadera incidencia de HSD tras punción dural es desconocida ya que la mayoría de los pacientes que presentan cefalea en este contexto, se tratan de forma conservadora sin más investigación. Aunque la literatura solo recoge casos aislados, se estima que la prevalencia de HSD secundario a anestesia neuroaxial es de 1/500.000 $1 / 1.000 .000$ de casos (4).

Según la Clasificación internacional de las cefaleas (ICDH-3BETA) de la 
International Headache Society, la cefalea postpunción dural (CPPD) es la que ocurre en los 5 días siguientes a una punción dural, causada por la fuga de LCR por el punto de punción, que normalmente se acompaña de rigidez cervical y síntomas auditivos. Suele remitir de forma espontánea en un plazo de 2 semanas o tras sellado del punto de fuga con un parche hemático. Característicamente, la CPPD, empeora con la bipedestación y sedestación y mejora con el decúbito.

Como tratamiento conservador de esta entidad, se recomienda: Apoyo psicológico; Medidas posturales, favoreciendo el decúbito supino $o$ decúbito prono; Hidratación agresiva, aunque no se ha podido demostrar evidencia científica que la apoye, la deshidratación sí podría agravar el caso y debería evitarse; Analgésicos no esteroideos y paracetamol pudiendo asociar un opioide débil; la Cafeína se usa como vasoconstrictor cerebral bloqueando receptores de adenosina y, algunos estudios han demostrado superioridad de la cafeína frente a placebo en el tratamiento de la CPPD, sin embargo el uso a dosis terapéuticas se puede asociar a un agravamiento de HTA previamente existente, toxicidad de sistema nervioso central y fibrilación auricular, por lo que no es una terapia sin complicaciones (8).

La formación de higromas subdurales quísticos es una complicación conocida, pero infrecuente, asociada a anestesia neuroaxial. Los higromas son colecciones de líquido xantocrómico o sanguinolento, que se desarrollan en el espacio subdural, a consecuencia de la creación de un espacio entre la aracnoides y la duramadre por hipotensión de LCR y desplazamiento caudal de estructuras, que causa extravasación de líquido desde los tejidos dañados. Se asocian frecuentemente a eventos traumáticos, pero pueden presentarse también en casos de atrofia cortical, alcoholismo, deshidratación o fístulas durales. La mayoría de los higromas se resuelven de manera espontánea cuando el cerebro se reexpande, sin embargo, en algunos casos, pueden causar sangrado.

Los mecanismos fisiopatológicos propuestos para la ocurrencia de la CPPD y del HSD son similares. Se produce pérdida de LCR por el agujero de punción, lo que causa una pérdida de volumen y disminución de presión a nivel del canal medular e intracraneal, provocando un desplazamiento caudal de la médula y cerebro, con tracción de estructuras sensibles al dolor, originándose así la cefalea. El drenaje venoso del cerebro se realiza a través de venas puente, que se dirigen a los senos durales. La porción más fina de estas venas puente se localiza a nivel del espacio subdural, por lo que es a ese nivel donde se produce el desgarro cuando se someten a tracción, desencadenándose el HSD.

De este modo, pueden presentarse 2 fases clínicas: inicialmente una cefalea con claro componente postural, debido a la hipotensión de LCR y, posteriormente un incremento de la intensidad del dolor con pérdida del componente ortostático y refractariedad al tratamiento habitual de la CPPD. Además, es frecuente la asociación de algunos de los siguientes síntomas: vómitos, diplopía, alteración cognitiva o del nivel de conciencia, o síntomas neurológicos focales. El desarrollo de esta sintomatología puede surgir en un intervalo de tempo variable, entre días y semanas, tras la punción. Esta secuencia temporal de la sintomatología se observa en los dos casos presentados. Ante la sospecha, se recomienda la realización de una prueba de imagen diagnóstica. De entrada, no se aconseja realizar una nueva punción dural para 
análisis de LCR ya que podría empeorar los síntomas.

Amorim et al (9), realizaron una revisión de 35 casos de HSD postanestesia neuroaxial e identificaron como factores de riesgo para esta complicación, el embarazo, realización de punciones múltiples, deshidratación, uso de anticoagulantes, presencia de anormalidades vasculares intracraneales $\mathrm{y}$ atrofia cerebral.

El tratamiento de estas complicaciones puede ser médico o quirúrgico, dependiendo del tamaño del hematoma o severidad de los síntomas. Los hematomas pequeños con mejoría gradual de los síntomas suelen responder a tratamiento médico. Sin embargo, los pacientes con deterioro neurológico $\quad \mathrm{o}$ importante desplazamiento de estructuras en pruebas de imagen pueden requerir drenaje quirúrgico urgente. En el primer caso, la paciente no presenta importante afectación radiológica, pese a desarrollar clínica neurológica, por lo que se decide manejo conservador. En el segundo caso, la paciente permanece sin focalidad neurológica ni afectación del nivel de conciencia, sin embargo, ante los hallazgos radiológicos de desplazamiento de línea media e incipiente herniación subfalciana, se opta por tratamiento quirúrgico urgente. Es de reseñar que tras el diagnóstico de un HSD, se debe solicitar la valoración urgente por parte de neurocirugía.

Este trabajo tiene como objetivo, aumentar la conciencia de los profesionales ante la posibilidad de aparición de este tipo de complicaciones ante una punción dural accidental durante una técnica de analgesia epidural de parto, ya que son fundamentales el diagnóstico $\mathrm{y}$ tratamiento precoz de estas entidades. En conclusión, la presencia de cefalea intensa, progresiva, con alteraciones en el componente postural, sin mejoría clara o con empeoramiento de los síntomas o asociación de síntomas neurológicos, debe considerarse una señal de alarma para sospechar una complicación intracraneal. Esta situación, aunque infrecuente, puede tener consecuencias catastróficas $y$ potencialmente fatales o producir un daño neurológico permanente.

\section{Bibliografía}

1. Cuypers V, Van de Velde V, Devroe S. Intracanial subdural haematoma following neuroaxial anaesthesia in the obstetric population: a literatura review with analysis of 56 reported cases. Int J Obstet Anesth. 2016; 25 : 58-65 ( $\underline{\text { PubMed }})$

2. Nepomuceno R, Herd A. Bilateral Subdural Hematoma after Inadvertent Dural Puncture During Epidural Analgesia. J Emerg Med. 2013; 44:227-230

3. Verdú MT, Alonso B, Burguillos S, Martinez-Lage JF. Postpartum Subdural Hygroma after Epidural Analgesia. Anesthesiology. 1999; 91: 867-9 (ubMed) (HTML)

4. Vaughan DJ, Stirrup CA, Robinson PN. Cranial subdural hematoma associated with dural pucture in labor. Br J Anaesth. 2000; 84 (4):518-20 (DF) (

5. Abbinante, Lauta E, Di venosa N, Ribezzi M, Colamaria A, Ciappett P. Acute subdural hematoma after combined epidural analgesia in labor. Minerva Anestesiol. 2010; 76:1091-194

6. Ramirez S, Gredilla E, Martinez B, Gilsanz F. Hematoma subdural bilateral secundário a punción dural acidental. Rev Bras Anestesiol. 2015; 65(4):306-309 ( $\underline{\text { PDF })}$

7. Reina MA, López A, Benito-León J, Pulido $P$, María F. Hematoma subdural intracraneal y espinal, una complicación poco común de la anestesia epidural y subaracnóidea. Rev. Esp. Anestesiol. Reanim. 2004; 51: 28-39 (PDF)

8. López Correa T, Garzón Sánchez JC, Sánchez Montero FJ, Muriel Villoria C. Cefalea postpunción dural en obstetricia. Rev. Esp. Anestesiol. Reanim. 2011; 58:563-573 (PDF) 
9. Amorim JA, Remigio D, Filho O, Barros MA, Carvalho VN, Valença M. Hematoma subdural intracraneal postanestesia subaracnoidea: relato de dos casos y revisión de 33 casos de la literatura. Rev. Bras. Anestesiol. 2010; 60(6): 624-629
Correspondencia al autor

Rita Veloso de Sousa rveloso.sou@gmail.com MIR Anestesiología y Reanimación. Hospital Universitario San Cecilio, Granada

Aceptado para blog en mayo de 2018 . 